\title{
Bubble characteristics in a bubbling fluidized bed with a rotating distributor
}

\author{
C. Sobrino ${ }^{\text {a,* }}$, A. Acosta-Iborra ${ }^{\text {a }}$, D. Santana ${ }^{\text {a }}$, M. de Vega ${ }^{\text {a }}$ \\ ${ }^{a}$ Energy Systems Engineering Group (ISE), Department of Thermal and Fluid \\ Engineering, Universidad Carlos III de Madrid
}

\begin{abstract}
In this paper the effect of a novel rotating distributor for fluidized beds on the bubble size is studied. The distributor is a perforated plate that rotates around the vertical axis of the column.

The formation of the bubbles from the rotating distributor is theoretically analyzed. The pierced length of the bubbles ascending in the bed were measured using optical probes. The probability distribution of bubble diameter was inferred from these experimental measurements using the maximum entropy method. The radial profile of the bubble diameter is presented for the static and rotating configurations at different gas velocities. The frequency of bubble passage and the distribution of bubbles in the cross section of the bed bed are also reported. Results were finally shown for different heights above the distributor.

A radial decrease in the bubble size when the distributor rotates is found. The bubble growth with the bed height is also lower in the rotating case.
\end{abstract}

Key words: Fluidized bed, Rotating distributor, Bubble size distribution, Optical probes.

\section{Introduction}

Gas bubbles present in most gas-solids beds can cause both chemical and mechanical difficulties. For instance, in gas-solids reactions, some of the gas in the bubbles may by-pass the particles altogether with little contact with the

\footnotetext{
* Corresponding author

Email address: csobrino@ing.uc3m.es

Tel.: +34916248884; Fax: +34916249430 (C. Sobrino).
} 
bed solids, thus, the overall efficiency of contact is lowered (Davidson and Harrison, 1963). However bubbles have also advantageous effects since they are responsible for the gas solids mixing and gas circulation and thus they play an important role in chemical reactions in fluidized beds. Therefore, controlling the bubble size to avoid large and fast bubbles that bypass the bed and increase the elutriation is an important task.

Bubble characteristics such as bubble size, shape or ascending velocity are important parameters that have been extensively studied in the past (Ghadiri et al., 1988; Dent et al., 1989; Werther and Molerus, 1973; Werther, 1974; Ramaya et al., 1993). Different techniques have been developed to study the bubble properties in fluidized beds, some of them reviewed by Cheremisinoff (1986); Yates and Simons (1994) and Werther (1999). One of the most popular techniques is the use of optical probes. They have been widely applied for the measurement of particle velocity and particle concentration in threedimensional fluidized beds and to a lesser extent to detect the passage of bubbles and measure their main parameters (Ishida and Shirai, 1980; Hatano and Ishida, 1981; Schweitzer et al., 2001; Glicksman et al., 1987).

The interpretation of the signals from dual submersible probes for bubble size determination is difficult as they give information on pierced length rather than characteristic diameter and this conversion is not straightforward. An extended review of the different methods employed for this conversion can be found in Santana et al. (2006).

Several methods have been used to improve the quality of fluidization. Vibration (Mawatari et al., 2003) and agitation (Kim and Han, 2006) have been used in some designs to avoid unwanted phenomena as channeling or agglomeration in the fluidization of cohesive fine particles. With the same aim Quevedo et al. (2006) employed a fluidized bed which consisted of a cylinder that acted as the gas distributor and rotated along its horizontal axis. On the other hand, flow pulsation (Köksal and Vural, 1998) has been studied as a way to control the bubble size. Other attempts consist on the design of special distributors. Swirling fluidizing patterns generated by different distributor designs (Chyang and Lin, 2002; Sreenivasan and Ragahavan, 2002) or the tangential injection of gas proved to increase the lateral mixing of solids giving a substantial improvement over the predominant axial flow pattern in conventional fluidized beds. The injection of secondary air with a swirl has been also successfully tested to intensify the lateral dispersion of particles in circulating fluidized beds (Ran et al., 2001). De Wilde and de Broqueville (2007) presented a new concept of rotating fluidized bed where the solids experimented a radially outwards centrifugal force cause by the tangential injection of the fluidization gas via multiple gas inlet in the outer cylindrical wall of the fluidization chamber. However, none of these works give information on the behavior of the bubbles. Coppens and van Ommen (2003) introduced three possibilities to structure chaotic fluidized beds: applying an $\mathrm{AC}$ electric field, introducing part of the gas via a fractal injector and oscillating the gas flow. Continuing this research, this group group published another work (van Ommen et al., 
2007) where besides the former methods the optimization of the particle size was proposed. In this work the size of the bubble was experimentally determined in 2D fluidized beds.

In this work the characteristics of the bubbles in a bubbling fluidized bed with a vertical axis rotating distributor are studied. This novel design was proposed by Sobrino et al. (2008) who reported a decrease of the minimum fluidization velocity, $U_{m f}$, when the rotational speed of the distributor increased. They also found that for low excess gas conditions, the energy of the power spectra of the pressure fluctuation was lower for the rotating distributor than for the static configuration.

In this work, the pierced length of the bubbles ascending in the bed for the two type of distributors was measured using optical probes (Vázquez et al., 2007). The probability density function of the bubble diameter was inferred from these experimental measurements using the methodology based on the maximum entropy method proposed by Sobrino (2008); Sobrino et al. (2009). The effect of the rotation on the bubble flow pattern was also studied.

\section{Bubble formation model}

Bubbles in a fluidized bed rise as if they were in an ordinary liquid of small viscosity and zero surface tension (Davidson and Harrison, 1963). The bubble and particulate phases of a fluidized bed appear to be analogous to the gas and liquid phases in a liquid-gas system. When air is blown steadily through an orifice into a liquid of small viscosity at intermediate flow-rates, a more or less regular train of bubbles is formed. The mechanism of bubble formation at the distributor orifices in fluidized beds has been extensively studied. Harrison and Leung (1961) found good agreement between experiments on bubble formation at an orifice in air-fluidized beds and in air-water systems. In this section, the influence of the rotational speed on the size of bubbles formed at the orifices of a rotating distributor, on the basis of a simplified model, will be studied. The frequency and size of bubbles in fluidized beds at intermediate flow-rates is governed mainly by a balance between buoyancy forces and inertial forces (Davidson and Harrison, 1963). Thus, the flow-rate in the emulsion phase can be neglected. In the present study the perforated plate where bubbles are formed rotates and thus the centrifugal force acting on the bubble when it detaches form the distributor plays also a role. The viscous drag is considered negligible, provided the low viscosity of fluidized beds. The magnitude of the buoyancy force, $F_{b}$ and the centrifugal force, $F_{c}$, can be given as:

$$
\begin{aligned}
& F_{b}=\rho_{b u l k} V g \\
& F_{c}=\frac{1}{2} \rho_{b u l k} V \omega^{2} r
\end{aligned}
$$


Where $\rho_{\text {bulk }}=\left[\rho_{p}(1-\epsilon)+\rho_{f} \epsilon\right]$ is the bulk density of the particulate phase, $V$ is the bubble volume at time $t, \omega$ is the rotational speed of the distributor and $r$ the radial coordinate. The bubble motion at detachment is defined as the balance of the buoyancy and centrifugal forces against the rate of change of momentum of the particle phase surrounding the bubble. The inertia of the air within the bubble can be neglected. Therefore, for the forming bubble the momentum at any instant is:

$$
M=\frac{1}{2} \rho_{b u l k} V u_{o}
$$

where $u_{o}$ is the velocity of the center of the ascending bubble.

It has to be noted that when a sphere moves in an inviscid fluid, with no separation of the flow, the effective mass added to the sphere by the surrounding fluid is half the displaced mass. This mass has been introduced in Eq. (2) and (3).

The buoyancy and centrifugal forces have perpendicular directions and hence the equation of the motion of the bubble is

$$
\sqrt{\left(\rho_{\text {bulk }} V g\right)^{2}+\left(\frac{1}{2} \rho_{b u l k} V \omega^{2} r\right)^{2}}=\frac{d}{d t}\left(\frac{1}{2} \rho_{\text {bulk }} V u_{o}\right)
$$

Besides, the bubble volume growth rate is:

$$
\frac{d V}{d t}=q
$$

Where $q$ denotes the volumetric flow rate through a hole of the distributor that goes into the bubble,

$$
q=\left(U-U_{m f}\right) A_{o}
$$

where $A_{o}$ is the area of the distributor per number of holes $\left(A / N_{o}\right), U$ is the superficial gas velocity and $U_{m f}$ is the minimum fluidization velocity. Other authors include a term accounting for the gas flowing through the bubble boundary in the calculation of the gas flow through the orifice (Vakhshouri and Grace, 2008), however for high static bed heights $\left(r_{b}<<H_{0}\right)$ or high distributor to bed pressure drops $\left(\Delta P_{d} / \Delta P_{b}\right)$, the pressure variation during bubble formation is small, and constant flow condition may be satisfied.

The volume of the bubble is $V=\frac{4}{3} \pi r_{b}^{3}$, where $r_{b}$ is the radius of the bubble. Therefore,

$$
\frac{d r_{b}}{d t}=\frac{q}{4 \pi r_{b}^{2}}
$$

Assuming that, at detachment, the velocity of the bubble center, $u_{o}$, is equal 
to the growth rate of the bubble $d r_{b} / d t$ (Fujikawa et al., 2003; Vakhshouri and Grace, 2008) and introducing Eq. (7) in the forces balance (4) gives,

$$
\sqrt{\left(\rho_{b u l k} V g\right)^{2}+\left(\frac{1}{2} \rho_{b u l k} V \omega^{2} r\right)^{2}}=\frac{\rho_{b u l k} q^{2}}{24 \pi r_{b d}^{2}}
$$

From this equation and using Eq. (6) the bubble radius at the detachment can be obtained as

$$
r_{b d}=\left[\frac{\left[\left(U-U_{m f}\right) A_{o}\right]^{2}}{32 \pi^{2} \sqrt{g^{2}+\left(\frac{1}{2} \omega^{2} r\right)^{2}}}\right]^{1 / 5}
$$

It can be seen in the above equation that the centrifugal acceleration $\frac{1}{2} \omega^{2} r$ promotes the decrease of the bubble size when it is formed at the distributor. Fig. 1 shows the expected bubble radius at detachment for the rotating distributor as a function of the radial position calculated using Eq. (9), for two different rotational speeds. The radius has been non-dimensionalized with the bubble radius at the center of the plate, where the centrifugal acceleration $\omega^{2} r$ is zero. It can be seen that for a given excess of gas, the rotation makes the bubble size to decrease. This decrease is higher at higher $r$ or higher rotational speeds since the centrifugal acceleration increases with $r$ and $\omega$.

[Fig. 1 about here.]

\section{Experimental}

Bubble pierced lengths were measured in the bubbling fluidized bed with the rotating distributor using optical probes. The column had $0.193 \mathrm{~m}$ inner diameter and $0.8 \mathrm{~m}$ height and was filled with Geldart B silica sand particles with a mean diameter of $680 \mu \mathrm{m}$ and a density of $2632.5 \mathrm{~kg} / \mathrm{m}^{3}$. The settled bed height was $22 \mathrm{~cm}$. The rotating distributor was a perforated plate with an open area ratio of $1 \%$ and the holes were laid out in an hexagonal pitch of $15 \mathrm{~mm}$. The distributor to bed pressure drop ratio for a fluidizing velocity $U$ $=0.4 \mathrm{~m} / \mathrm{s}$ was $\Delta P_{d} / \Delta P_{b}=0.5$. The distributor could rotate in the horizontal plane as it was coupled to the shaft of an AC electric motor. A detail of the mechanical assembly of the rotating distribution and a 3D schematics of the set-up can be seen in Fig. 2.

[Fig. 2 about here.]

Experiments for the distributor without rotation (static configuration) and the distributor rotating at $100 \mathrm{rpm}$ were compared. Optical probe measurements 
had a duration of $10 \mathrm{~min}$ and the sampling frequency was $500 \mathrm{~Hz}$. The data were recorded with a 12 bits data acquisition board (ICP DAS PCI-1802H) assembled in a PC. Further details on the experimental set-up can be found in Sobrino et al. (2008)

The optical probes developed for this study are based on backscattering principle. The optical fiber probes (OFP) were made of two standard step-index plastic optical fibers (emitter and receiver fibers) embedded in a metallic coil of $3 \mathrm{~mm}$ external diameter. The fibers had a diameter of $1 \mathrm{~mm}$ with 0.22 $\mathrm{dB} / \mathrm{m}$ attenuation and $0.47 \mathrm{NA}$. The propagation delay constant of the fiber was $5 \mathrm{~ns} / \mathrm{m}$ and the whole probe had a length of about $1 \mathrm{~m}$. The emitter fiber was illuminated by a $650 \mathrm{~nm}$ laserdiode with a maximum power of $10 \mathrm{~mW}$ (Roithner s6510mg) and a phototransistor was used at the reception, both encapsulated in ST connectors. A 50/50 passive splitter is used for splitting the optical power into the emitter fibers of both probes. In order to measure the pierced length and velocity of the ascending bubbles, these two probes were placed one above the other $1 \mathrm{~cm}$ apart.

Measurements were carried out at different radial positions and different heights above the distributor. The bubble velocity was obtained calculating the cross correlation of the signal recorded from the upper and the lower probes. The bubble pierced length was determined from the time during which one of the probe is immersed into the bubble. The probability density function of the bubble size was estimated from the sample of bubble pierced lengths measured applying the maximum entropy method. This method is used to obtain the probability density function of a variable (in this case the bubble diameter) provided the distribution is subjected to a certain number of constrains that are known (the geometric moments of the diameter distribution). As the parameter measured using optical probes in fluidized bed is not directly the bubble diameter but the bubble pierced length, the raw moments of the bubble diameter distribution have to be estimated from the bubble pierced lengths deducing the equations that relate both magnitudes. The estimation of this relation entails some assumptions: the bubbles are assumed to rise randomly distributed in a horizontal circular surface containing the probe (Werther, 1974) and the ascending velocity of the bubble is considered vertical. This last hypothesis has been evaluated to have a negligible effect on the bubble size distribution estimation (Santana et al., 2006). It has also been considered that the minimum bubble pierced length that is possible to measure accurately using intrusive probes is equal to the distance between probes, which is $1 \mathrm{~cm}$ in this case (Sobrino et al., 2008). Finally, the geometry of the bubbles have been modeled as a truncated spheroid, which is the most general shape adopted by the bubbles in a fluidized bed. A detailed explanation of the equations applied to estimate the bubble size distribution using the maximum entropy method and the signal analysis carried out to measure the velocity and pierced length of the ascending bubbles can be found elsewhere (Sobrino, 2008; Sobrino et al., 2009).

The effect of the rotation on the bubble size and bubble flow pattern was an- 
alyzed from measurements carried out at different axial and radial positions.

\section{Results and discussion}

Sobrino et al. (2008) studied the global characteristics of the flow in the fluidized bed with rotating distributor employed in this study. They found that the minimum fluidization velocity, $U_{m f}$, decreases when the rotational speed increases. The minimum fluidization velocity for the static configuration is $U_{m f, 0} \sim 0.4 \mathrm{~m} / \mathrm{s}$ while for the distributor rotating at $100 \mathrm{rpm}, U_{m f, 100} \sim 0.3$ $\mathrm{m} / \mathrm{s}$. In this work experiments were carried out for three different cases:

(a) The static distributor with a superficial gas velocity $U=0.57 \mathrm{~m} / \mathrm{s}(U-$ $U_{m f, 0}=0.17 \mathrm{~m} / \mathrm{s}$ ).

(b) The distributor rotating at $n=100 \mathrm{rpm}$ with a superficial gas velocity $U=0.57 \mathrm{~m} / \mathrm{s}\left(U-U_{m f, 100}=0.27 \mathrm{~m} / \mathrm{s}\right)$.

(c) The distributor rotating at $n=100 \mathrm{rpm}$ with a superficial gas velocity $U$ $=0.46 \mathrm{~m} / \mathrm{s}\left(U-U_{m f, 100}=0.16 \mathrm{~m} / \mathrm{s}\right)$.

The comparison between cases (a) and (b) shows the effect of the rotation for equal gas velocities. Since $U_{m f}$ is lower for the rotating distributor, the excess gas is higher in this case (case (b)) than for the static distributor (case (a)). A comparison between cases (a) and (c) shows the effect of rotation for similar excess gas conditions.

\subsection{Radial profile of bubble size}

The theory of bubble formation explained in the previous section pointed out the influence of the rotation on the bubble diameter at its detachment. It was shown that the centrifugal acceleration promotes the decrease of the bubble size and therefore, this effect is more pronounced at higher radial positions. Bubble pierced lengths were measured along the bed radius at $7.5 \mathrm{~cm}$ above the distributor. Three different cases were studied corresponding to the experimental conditions described at the beginning of this section.

Fig. 3 shows the probability density function (PDF) of the bubble diameter at different radial positions for the three cases studied. These distributions were obtained from the experimental measurement of the bubble pierced length, applying the method based on the maximum entropy principle proposed by Sobrino et al. (2008). Fig. 3(a) shows the results for the static configuration (case (a)) and Fig. 3(b) and 3(c) show the results for the rotating configuration for the same gas velocity and excess gas respectively as in the static case. So- 
brino (2008); Sobrino et al. (2009) showed that there is a minimum bubble size that it is possible to measure accurately using intrusive probes, due to their finite size. Therefore, the distributions in Fig. 3 are the distributions of diameters larger than $D_{v, \text { min }}$, where $D_{v, \text { min }}$ is the diameter of the smallest bubble that can be measured with the probes. Sobrino (2008); Sobrino et al. (2009) showed that the smallest bubbles that can be measured with this system have a diameter of approximately the distance between probes $(s=1 \mathrm{~cm})$. Fig 3 shows that for values of $r / R$ larger than 0.4 , the bubble size distributions for the rotating and static cases at the same excess gas differ: when the distributor rotates the peak is displaced to lower $D_{v}$ and the variance also diminishes (Fig. 3(c)). For larger excess gas the rotation effect tends to disappear (Fig $3(\mathrm{~b}))$.

[Fig. 3 about here.]

The mean values of the distributions shown in Fig. 3 are plotted in Fig. 4, as a function of the radial position. At the higher velocity $(U=0.57 \mathrm{~m} / \mathrm{s})$ similar profiles are shown for the static and rotating configurations. The bubble diameter for the rotating distributor is only slightly larger than for the static case, even though the excess gas is much higher. When similar excess gas conditions are compared, it can be observed that quite smaller bubbles are found close to the bed wall for the rotating case. At higher $r$, the centrifugal acceleration is higher and then, there is a marked effect on bubble size. This is in agreement with the behavior predicted from the forces balance shown in Fig. 1 where smaller bubbles were shown to be formed close to the wall. The unclear tendency close to the axis, may be explained by the presence of the mechanical coupling between the distributor and the engine shaft, which makes the distributor axis a singular point

Sobrino et al. (2008) compared the power spectra of the pressure fluctuations measured in the bed working with the rotating and with the static distributor. The dominant frequency of the power spectrum of the pressure fluctuations in a fluidized bed depends mainly on the characteristic frequency of exploding bubbles in the bed surface. Moreover, the magnitude of the peak at this dominant frequency is related with the size of the bubbles in the bed (Sobrino et al., 2008b). Sobrino et al. (2008) reported a smaller peak in the power spectrum for the distributor rotating at $100 \mathrm{rpm}$ when the excess gas was low. They found similar power spectra for the static and rotating configuration at higher excess gas $\left(U / U_{m f} \sim 2\right)$. These results are in accordance with those found in this work where it was shown that, except for radial positions close to the bed axis, the mean pierced lengths are smaller for the rotating distributor.

[Fig. 4 about here.] 
[Fig. 5 about here.]

Fig. 5 shows the number of bubbles that are detected at each radial positions for each of the three cases described above. It can be seen that there are only a few bubbles ascending at the bed axis for the static distributor. This is a consequence of the holes layout and the absence of holes in this area. However, when the plate rotates, more bubbles appear in the bed axis since the rotation promotes the more homogeneous distribution of bubbles in the bed. Moreover, it was shown in Fig. 4 that, when comparing the static and rotating distributors at the same superficial gas velocity, both exhibit similar bubble sizes, even if the excess gas is higher in the rotating case. Observing the two cases at $U=0.57 \mathrm{~m} / \mathrm{s}$ in Fig. 5 , it can be seen that the ratio between the total number of bubbles (the addition of the bubbles detected at the different radial positions) for the static (5164 bubbles) and the rotating case (9068 bubbles) is very similar to the ratio between the excess gas $\left(U-U_{m f}\right)_{\text {stat }} /\left(U-U_{m f}\right)_{\text {rot }} \sim$ 0.6. Since the size of the bubbles was shown to be similar for the static and rotating configuration it can be concluded that the higher excess of gas in the rotating case is transported by a higher number of bubbles. As for the two cases at similar excess gas ratio, it can be seen that the sum of the number of bubbles detected at the different positions is approximately the same for both cases but they are distributed in a different manner along the bed surface.

\subsection{Height effect}

The change of the bubble size with the height in the bed has been studied carrying out measurements at different axial positions and a given radial position $\mathrm{r} / \mathrm{R}=0.8$. Experiments with the static and rotating distributor at the same gas velocity were compared. Probability distributions of the volume equivalent diameter at different heights in the bed are shown in Fig. 6. With the static distributor there are clear differences when measuring at different heights in the bed: an increase of the mode of $D_{v}$ and of the variance of the distribution are observed when the height increases. In the case of the rotating distributor more homogeneity is found all over the bed (at this radial position): the mode and the variance remains almost the same at different heights.

The mean values of these distributions are shown in Fig. 7 . It can be observed that the expected growth of the bubble size with distance above the distributor due to coalescence of bubbles (Darton et al., 1977) is only found for the static case. Bubble diameter using the rotating distributor barely increases as height increases. For heights larger than $10 \mathrm{~cm}$ the bubble size is similar for both distributors or even smaller for the rotating case even if the excess gas is larger in the rotating case. This effect could be explained by the fact that the bubble coalescence rate is lowered by the rotation of the distributor which 
may promote the break up of the preferential paths for the bubbles.

Measurements at other radial positions $(r / R=0.5$ and $r / R=0)$ and higher $z$ are shown in Fig. 8.

[Fig. 6 about here.]

[Fig. 7 about here.]

[Fig. 8 about here.]

It can be seen again, that at higher axial positions, when the amount of gas used to fluidize both, the static and the rotating distributor beds, is the same, even if the excess gas is higher, the bubble diameter is lower for the rotating distributor. The number of bubbles detected for each case is shown in the figure. It is seen that more bubbles are found in the rotating case and even if the bed is operating with more excess gas the bubbles are smaller: in the rotating bed higher excess gas can be handled with a larger number of bubbles of smaller size.

\section{Conclusions}

The size, spatial distribution and frequency of the bubbles found in a fluidized bed for configurations involving a static and a rotating distributor were compared. The motion equation of the bubbles formed at the distributor points out that the centrifugal acceleration imparted by the rotation causes the decrease of the initial bubble radius. This theoretical result is corroborated by experimental measurement of bubble size in the bed, near the distributor.

Smaller bubbles were found for the rotating distributor when the excess gas for the static and rotating configuration was similar. The bubble size radial profile indicates that when the distributor rotates, the diameter of the bubbles close to the bed walls is smaller, the centrifugal acceleration being higher. The distributor rotation also promotes a more homogeneous distribution of the bubbles over the bed surface. The study of the bubble size at different heights in the bed shows that for the same gas velocity bubble diameter is higher when the distributor rotates than for the static distributor. This was expected since the minimum fluidization velocity is much lower for a rotational speed of $100 \mathrm{rpm}$. However the difference in bubble diameter is lost at higher axial positions and even smaller bubbles are found for the rotating case at heights over about $11 \mathrm{~cm}$ above the distributor. This may be due to the rupture of the preferential paths by the effect of the rotation what makes bubble coalesce events less frequent. 


\section{Notations}

A Cross-sectional area of the bed $\left(\mathrm{m}^{2}\right)$

$A_{o} \quad$ Distributor area per number of holes $\left(\mathrm{m}^{2}\right)$

$D$ Bed diameter $(m)$

$D_{v} \quad$ Bubble volume equivalent diameter $(m)$

$D_{v, \min } \quad$ Lower limit of $D_{v}$ distribution $(m)$

$F_{b} \quad$ Buoyancy force $(N)$

$F_{c} \quad$ Centrifugal Force $(N)$

$g$ Gravity acceleration $\left(\mathrm{m} / \mathrm{s}^{2}\right)$

$H_{0} \quad$ Static bed height $(m)$

$M \quad$ Momentum $(\mathrm{kgm} / \mathrm{s})$

$n$ Rotational speed of the distributor plate $(\mathrm{rpm})$

$n_{b}$ Number of bubbles detected by the probe

$N_{o} \quad$ Number of orifices in distributor plate (-)

$q$ Volumetric flow rate through a hole of the distributor $\left(\mathrm{m}^{3} / \mathrm{s}\right)$

$r$ Radial coordinate

$r_{b}$ Bubble radius $(m)$

$r_{b d}$ Bubble radius at detachment $(m)$

$R$ Bed radius $(m)$

$u_{o} \quad$ Velocity of the bubble center $(\mathrm{m} / \mathrm{s})$

$U$ Superficial gas velocity $(\mathrm{m} / \mathrm{s})$

$U_{m f} \quad$ Minimum fluidization velocity $(\mathrm{m} / \mathrm{s})$

$U_{m f, 0} \quad$ Minimum fluidization velocity for the static distributor plate $(\mathrm{m} / \mathrm{s})$

$U_{m f, 100}$ Minimum fluidization velocity for the distributor plate rotating at 100 $\operatorname{rpm}(\mathrm{m} / \mathrm{s})$

$V \quad$ Bubble volume $\left(m^{3}\right)$

$V_{v} \quad$ Volume of a sphere having the same diameter as the bubble $\left(\mathrm{m}^{3}\right)$

$z$ Height above the distributor $(m)$ 
$\omega=\frac{2 \pi n}{60}$ Angular velocity of the distributor plate $\left(s^{-1}\right)$

$\Delta P_{b} \quad$ Bed pressure drop $(P a)$

$\Delta P_{d} \quad$ Distributor pressure drop $(P a)$

epsilon Void fraction (-)

\section{References}

Cheremisinoff, N. P., 1986. Review of experimental methods for studying the hydrodynamics of gas-solid fluidized beds. Ind. Eng. Chem. Process Des. Dev 25, 329-351.

Chyang, C.-S., Lin, Y.-C., 2002. A study in the swirling fluidizing pattern. Journal of Chemical Engineering of Japan 35, 503-512.

Coppens, M. O., van Ommen, J. R., 2003. Structuring chaotic fluidized beds. Chemical Engineering Journal 96, 117-124.

Darton, R. C., LaNauze, R. D., Davidson, J. F., Harrison, D., 1977. Bubblegrowth due to coalescence in fluidized-beds. Transactions of the institution of chemical engineers 55, 274-280.

Davidson, J. F., Harrison, D., 1963. Fluidized particles. Cambridge University Press.

Dent, D., LaNauze, R. D., Joyce, T., Fulford, V., Peeler, P., 1989. Differential pressure measurements - their application to measuremets of fluidized bed combustion parameters. In: Proc. 10th Conf. Fluidized Bed Combustion. pp. 451-456.

De Wilde, J., de Broqueville, A., 2007. Rotating Fluidized Beds in a Static Geometry: Experimental Proof of Concept. AIChe Journal 53, 793-810.

Fujikawa, S., Zhang, R., Hayama, S., Peng, G., 2003. The control of microair-bubble generation by a rotational porous plate. International Journal of Multiphase Flow 29, 1221-1236.

Ghadiri, M., Carter, B., Rathbone, R. R., Clift, R., Medhurst, S. J., Rogers, E. A., Summerfield, I., 1988. Diagnosis of gas flow patterns in fluidised beds. In: Proc. 4th International Fluidized Bed Combustion Conference. Institute of Energy.

Glicksman, L. R., Lord, W. K., Sakagami, M., 1987. Bubble properties in large-particle fluidized beds. Chemical Engineering Science 42, 479-491.

Harrison, D., Leung, L. S., 1961. Bubble formation at an orifice in a fluidised bed. Trans. Instn. Chem. Engrs., Lond. 39, 409-414.

Hatano, H., Ishida, M., 1981. The entrainment of solid particles from a gassolid fluidized bed. Journal of Chemical Engineering of Japan 14, 306-311.

Ishida, M., Shirai, T., 1980. Measurement of the velocity and direction of flow of solid particles in a fluidized bed. Powder Technology 27, 1-6. 
Kim, J., Han, G. Y., 2006. Effect of agitation on fluidization characteristics of fine particles in a fludized bed. Powder Technology 166, 113-122.

Köksal, M., Vural, H., 1998. Bubble size control in a two- dimensional fluidized bed using a moving double plate distributor. Powder Technology 95, 205213.

Mawatari, Y., Tatemoto, Y., Noda, K., 2003. Prediction of minimum fluidization velocity for vibrated fluidized bed. Powder Technology 131, 66-70.

Quevedo, J., Pfeffer, R., Shen, Y., Dave, R., Nakamura, H., Watano, S., 2006. Fludization of Nanoaglomerates in a Rotating Fluidized Bed. AIChe Journal $52,2401-2412$.

Ramaya, A. V., Venkateshan, S. P., Kolar, A. K., 1993. Large particle fluidization studies witha differential pressure fluctuation record. In: Rubow, L. (Ed.), Proceedings of the 12th International Conference on Fluidized Bed Combustion. Vol. 2. ASME, pp. 877-885.

Ran, X., Wei, F., Zhanwen, W., Yong, J., 2001. Lateral solids dispersion in high-density riser with swirling air flow. Powder Technology 121, 123-130.

Santana, D., Rodríguez-Rodríguez, J., Almedros-Ibáñez, J. A., MartínezBazán, C., 2006. Characteristics lengths and maximum entropy estimation from probe signals in the ellipsoidal bubble regime. International Journal of Multiphase Flow 32, 1123-1139.

Schweitzer, J-M., B. J., Gauthier, T., 2001. Local gas hold-up measurements in fluidized bed and slurry bubble column. Chemical Engineering Science $56,1103-1110$.

Sobrino, C., 2008. Experimental study of a bubbling fluidized bed with a rotating distributor. Ph.D. thesis, Universidad Carlos III de Madrid. http://hdl.handle.net/10016/3576

Sobrino, C., Almedros-Ibáñez, J. A., Santana, D., de Vega, M., 2008. Fludization of group B particles with a rotating distributor. Powder Technology 181, 273-280.

Sobrino, C., Sánchez-Delgado, S., García-Hernando, N., de Vega, M., 2008. Standard deviation of absolute and differential pressure fluctuations in fluidized beds of group B particles. Chemical Engineering Research and Design, $86,1236-1242$.

Sobrino, C., Almedros-Ibáñez, J. A., Santana, D., Vázquez, C, de Vega, 2009. Maximum entropy estimation of the bubble size distribution in fluidized beds. Chemical Engineering Science, doi: 10.1016/j.ces.2009.01.046

Sreenivasan, B., Ragahavan, V. R., 2002. Hydrodynamics of a swirling fluidised bed. Chemical Engineering Science 41, 99-106.

Vakhshouri, K., Grace, J.R., 2008. Modelling of bubble formation at a submerged orifice in a gas-fludized bed, Chem Eng Res Des, doi:10.1016/j.cherd.2008.10.002

van Ommen, J. R., Nijenhuis, J., van den Bleek, C. M., Coppens, M. O., 2007. Four Ways to Introduce Structure in Fluidized Bed Reactors. Ind. Eng. Chem. Res. 46, 4236-4244.

Vázquez, C., Nombela, J. L., Sobrino, C., de Vega, M., Zubía, J., Montero, 
D. S., 2007. Plastic fiber-optic probes for characterizing fluidized beds in bubbling regime. Vol. 16th International Conference on Plastic Optical Fiber (POF).

Werther, J., 1974. Bubbles in gas fluidised beds - Part II. Trans. Instn. Chem. Engrs 52, 149-159.

Werther, J., 1999. Measurements techniques in fluidized beds. Powder Technology 2, 15-36.

Werther, J., Molerus, O., 1973. The local structure of gas fluidized beds -I. a statistically based measuring system. International Journal of Multiphase Flow 1, 103-122.

Yates, J. G., Simons, S. J. R., 1994. Experimental methods in fluidization research. International Journal of Multiphase Flow 20 Suppl., 297-330. 


\section{List of Figures}

1 Bubble radius at detachment for two different rotational speeds calculated using Eq. 9 for a gas distributor of $R=$ $0.193 \mathrm{~m}$.

2 (a) 3D schematics of the fluidized bed with the rotating distributor. (b) Mechanical assembly of the rotating distributor.

$3 \quad \mathrm{PDF}$ of volume equivalent diameter at different radial positions (a) Static distributor, $U=0.57 \mathrm{~m} / \mathrm{s}, U_{m f, 0}=0.4 \mathrm{~m} / \mathrm{s}$. (b) Rotating distributor $n=100 \mathrm{rpm}, U=0.57 \mathrm{~m} / \mathrm{s}, U_{m f, 100}=$ $0.3 \mathrm{~m} / \mathrm{s}$. (c) Rotating distributor $n=100 \mathrm{rpm}, U=0.46 \mathrm{~m} / \mathrm{s}$, $U_{m f, 100}=0.3 \mathrm{~m} / \mathrm{s} . H=22 \mathrm{~cm} . z=7.5 \mathrm{~cm}$.

4 Mean volume equivalent diameter against the radial position for the static and rotating configuration $(n=100 \mathrm{rpm})$ at different gas velocities and excess gas conditions. $H=22 \mathrm{~cm}$. $z=7.5 \mathrm{~cm} . U_{m f, 0}=0.4 \mathrm{~m} / \mathrm{s} . U_{m f, 100}=0.3 \mathrm{~m} / \mathrm{s}$.

$5 \quad$ Number of bubbles detected at different radial positions for the static and rotating configuration $(n=100 \mathrm{rpm})$ at different gas velocities and excess gas conditions. $H=22 \mathrm{~cm} . z=7.5$ cm. $U_{m f, 0}=0.4 \mathrm{~m} / \mathrm{s} . U_{m f, 100}=0.3 \mathrm{~m} / \mathrm{s}$.

$6 \quad \mathrm{PDF}$ of $D_{v}$ at different heights above the distributor. (a) Static distributor $U=0.57 \mathrm{~m} / \mathrm{s}, U_{m f, 0}=0.4 \mathrm{~m} / \mathrm{s}$. (b) Rotating distributor at $n=100 \mathrm{rpm} U=0.57 \mathrm{~m} / \mathrm{s} U_{m f, 100}=0.3 \mathrm{~m} / \mathrm{s}$. $\mathrm{r} / \mathrm{R}=0.8$.

7 Mean value of volume equivalent diameter, against the height above the distributor for the static and rotating configuration $(n=100 \mathrm{rpm})$ at the same gas velocity. $r / R=0.8 \mathrm{~cm}$.

$8 \quad \mathrm{PDF}$ of $D_{v}$ at $U=0.57 \mathrm{~m} / \mathrm{s}$ and $z=12.5 \mathrm{~cm}$ for the static $\left(U_{m f, 0}=0.4 \mathrm{~m} / \mathrm{s}\right)$ and rotating distributor $\left(U_{m f, 100}=0.3\right.$ $\mathrm{m} / \mathrm{s}$ ). (a) $\mathrm{r} / \mathrm{R}=0.5$ (b) $\mathrm{r} / \mathrm{R}=0$. The mean of the distribution, $D_{v}$ and the number of bubbles detected by the probe in a 10 min interval are indicated in the plots. 


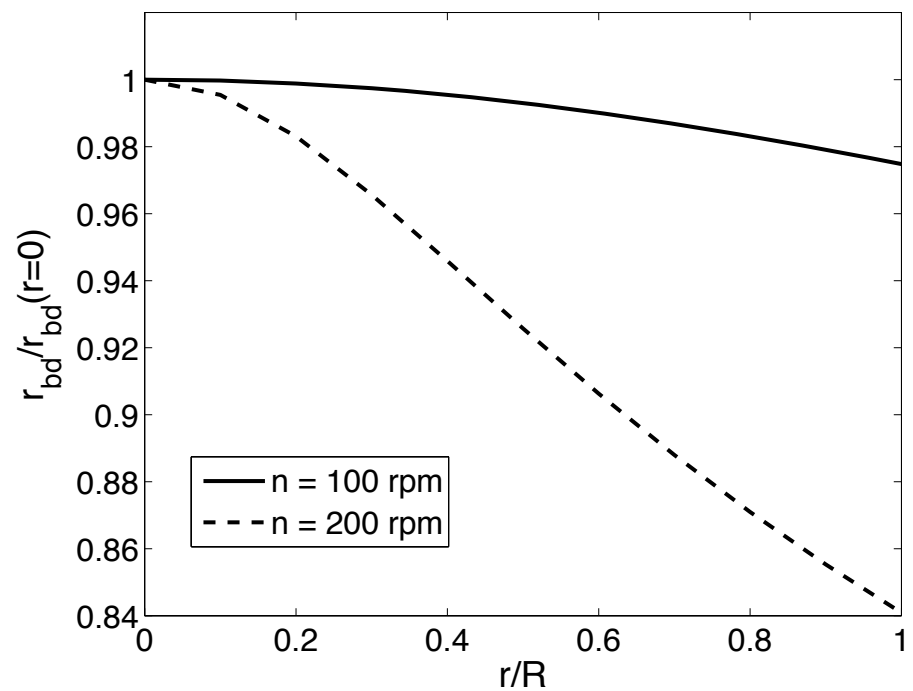

Fig. 1. Bubble radius at detachment for two different rotational speeds calculated using Eq. 9 for a gas distributor of $R=0.193 \mathrm{~m}$. 


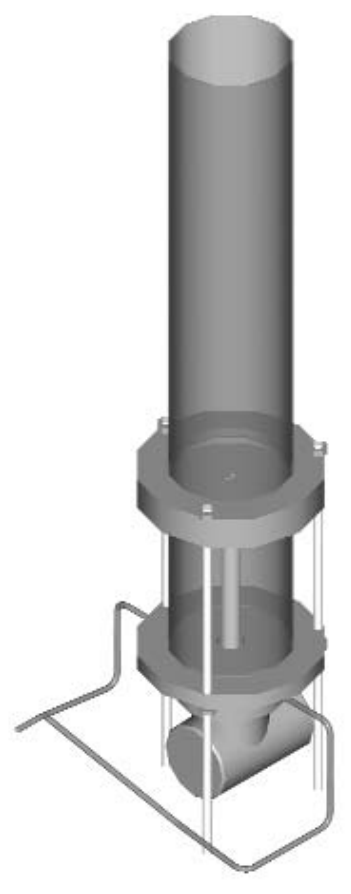

(a)

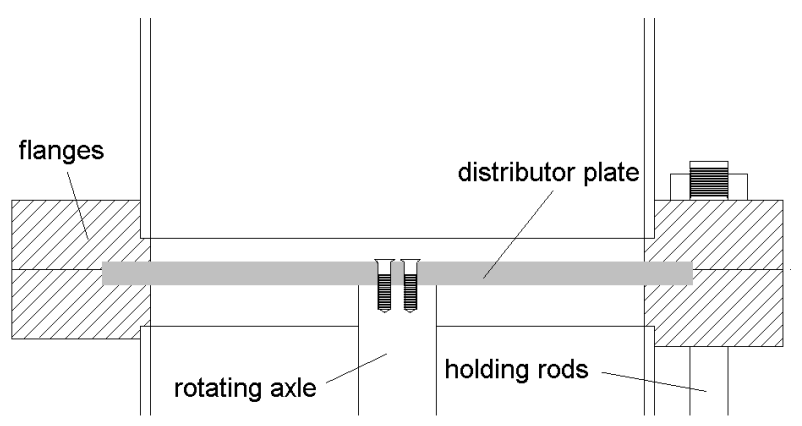

(b)

Fig. 2. (a) 3D schematics of the fluidized bed with the rotating distributor. (b) Mechanical assembly of the rotating distributor. 


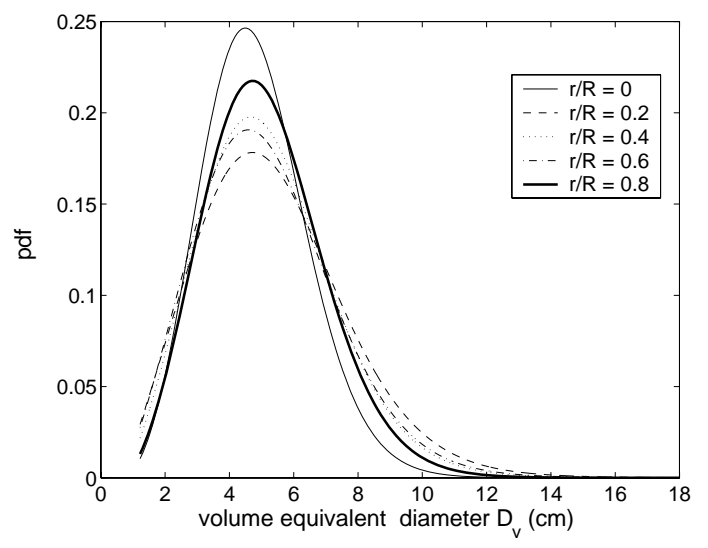

(a)

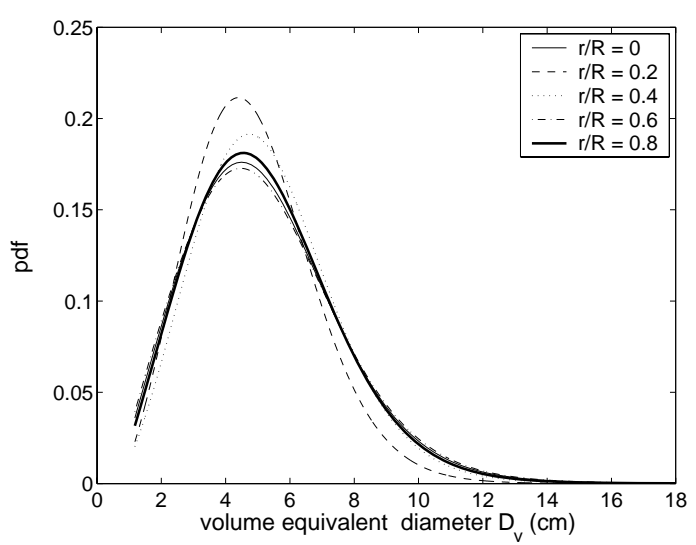

(b)

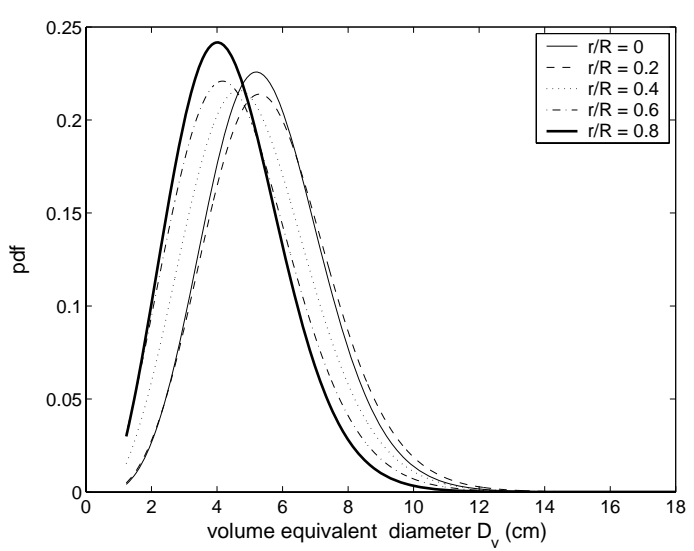

(c)

Fig. 3. PDF of volume equivalent diameter at different radial positions (a) Static distributor, $U=0.57 \mathrm{~m} / \mathrm{s}, U_{m f, 0}=0.4 \mathrm{~m} / \mathrm{s}$. (b) Rotating distributor $n=100 \mathrm{rpm}$, $U=0.57 \mathrm{~m} / \mathrm{s}, U_{m f, 100}=0.3 \mathrm{~m} / \mathrm{s}$. (c) Rotating distributor $n=100 \mathrm{rpm}, U=0.46$ $\mathrm{m} / \mathrm{s}, U_{m f, 100}=0.3 \mathrm{~m} / \mathrm{s} . H=22 \mathrm{~cm} . z=7.5 \mathrm{~cm}$. 


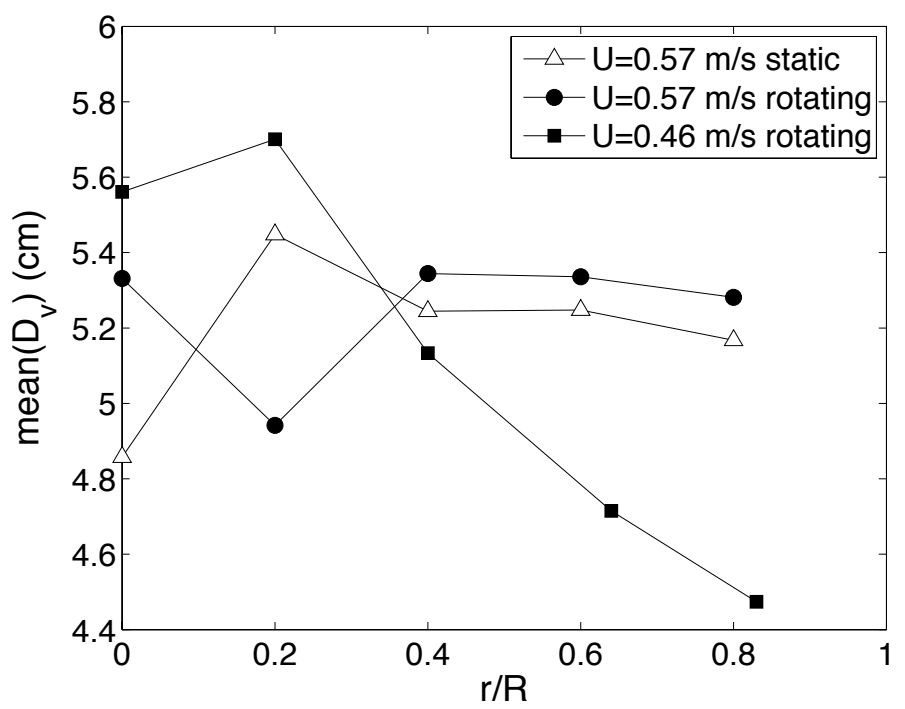

Fig. 4. Mean volume equivalent diameter against the radial position for the static and rotating configuration $(n=100 \mathrm{rpm})$ at different gas velocities and excess gas conditions. $H=22 \mathrm{~cm} . z=7.5 \mathrm{~cm} . U_{m f, 0}=0.4 \mathrm{~m} / \mathrm{s} . U_{m f, 100}=0.3 \mathrm{~m} / \mathrm{s}$. 


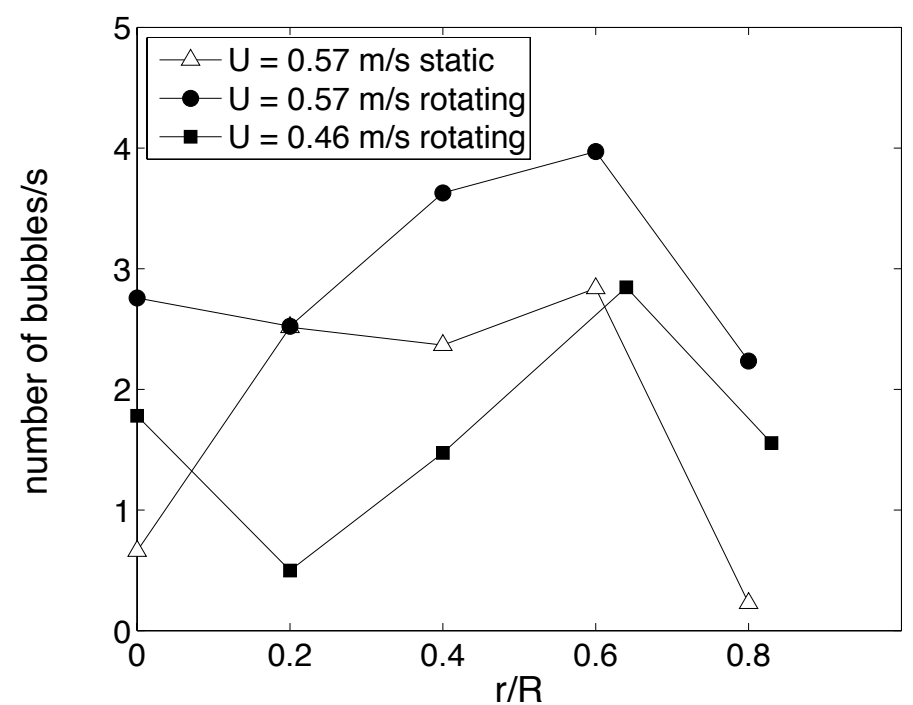

Fig. 5. Number of bubbles detected at different radial positions for the static and rotating configuration $(n=100 \mathrm{rpm})$ at different gas velocities and excess gas conditions. $H=22 \mathrm{~cm} . z=7.5 \mathrm{~cm} . U_{m f, 0}=0.4 \mathrm{~m} / \mathrm{s} . U_{m f, 100}=0.3 \mathrm{~m} / \mathrm{s}$. 


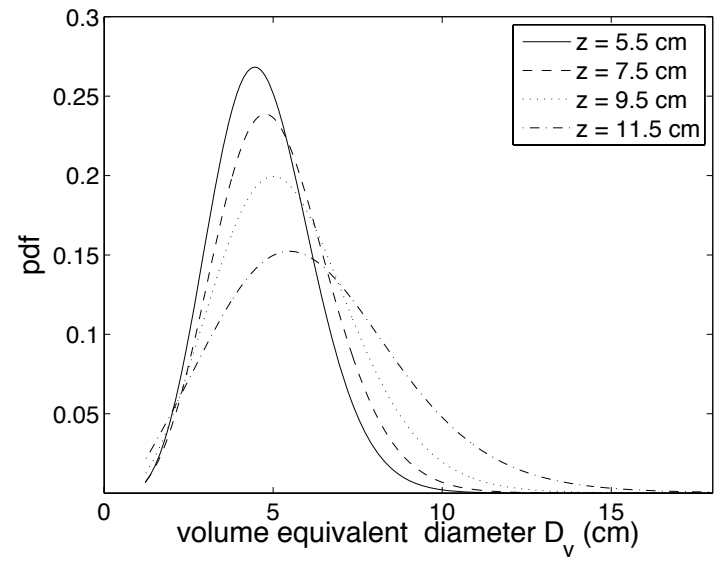

(a)

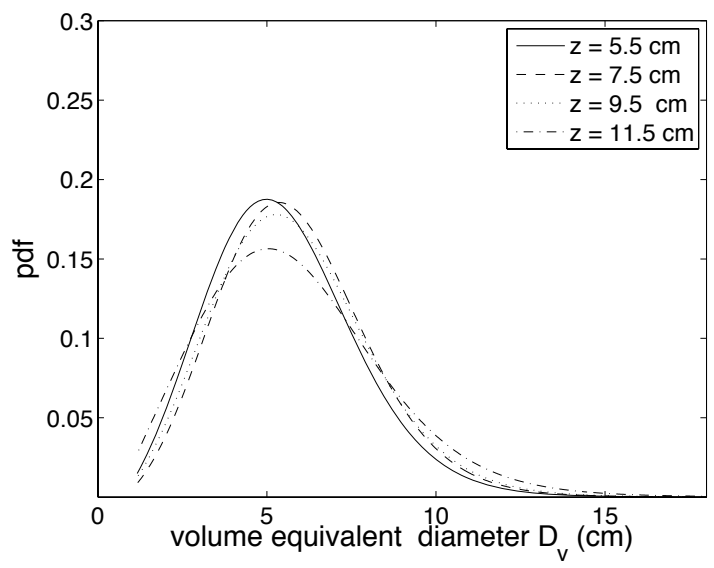

(b)

Fig. 6. PDF of $D_{v}$ at different heights above the distributor. (a) Static distributor $U=0.57 \mathrm{~m} / \mathrm{s}, U_{m f, 0}=0.4 \mathrm{~m} / \mathrm{s}$. (b) Rotating distributor at $n=100 \mathrm{rpm} U=0.57$ $\mathrm{m} / \mathrm{s} U_{m f, 100}=0.3 \mathrm{~m} / \mathrm{s} . \mathrm{r} / \mathrm{R}=0.8$. 


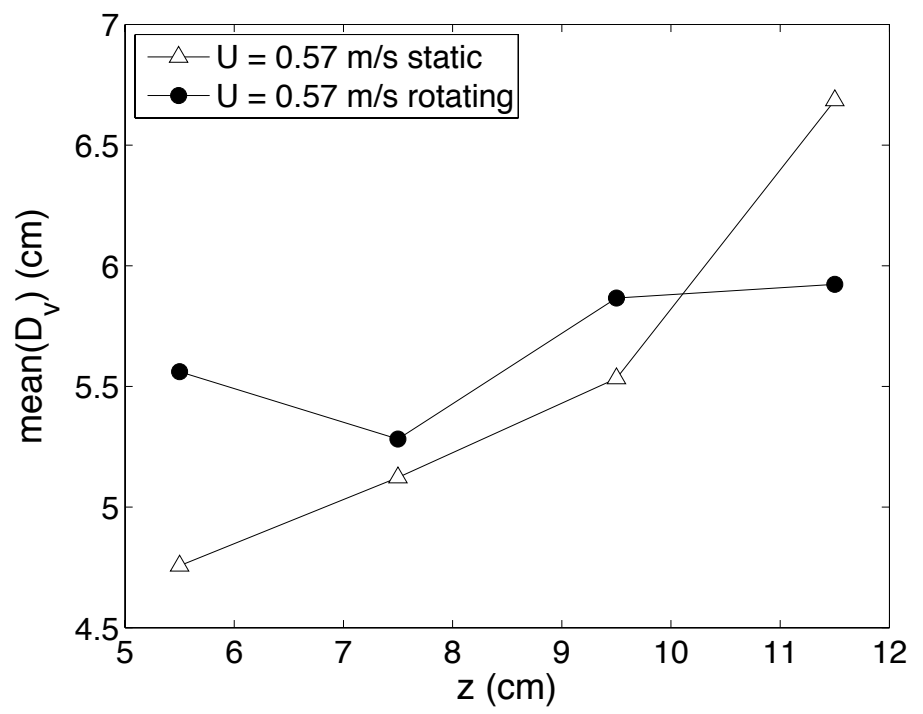

Fig. 7. Mean value of volume equivalent diameter, against the height above the distributor for the static and rotating configuration $(n=100 \mathrm{rpm})$ at the same gas velocity. $r / R=0.8 \mathrm{~cm}$. 


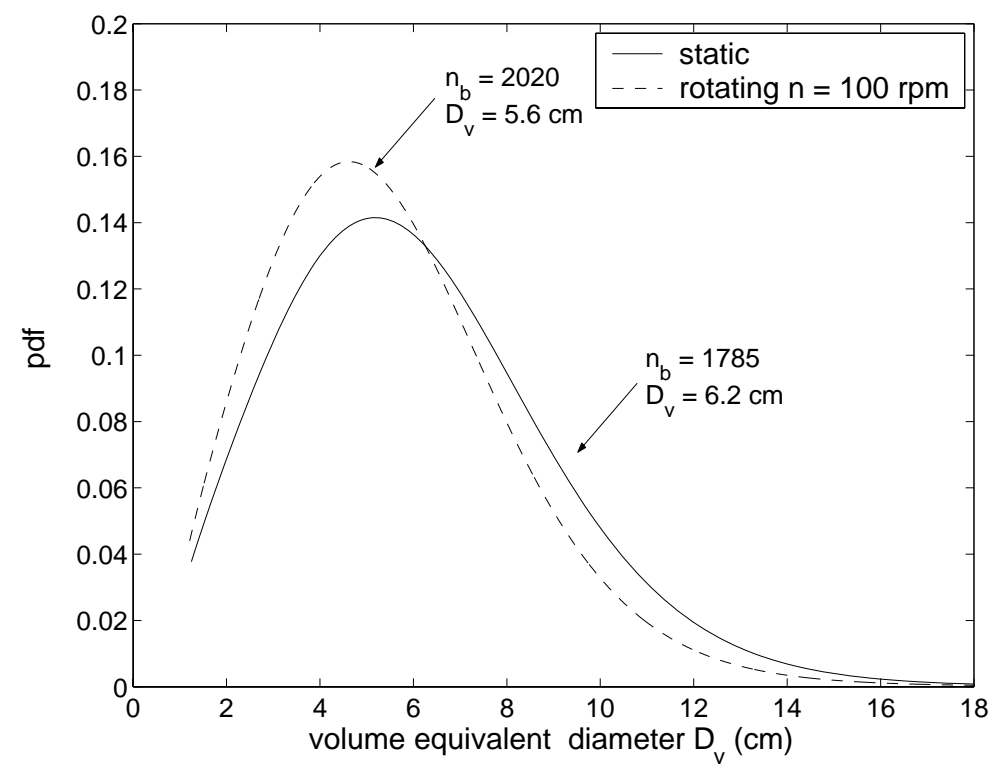

(a)

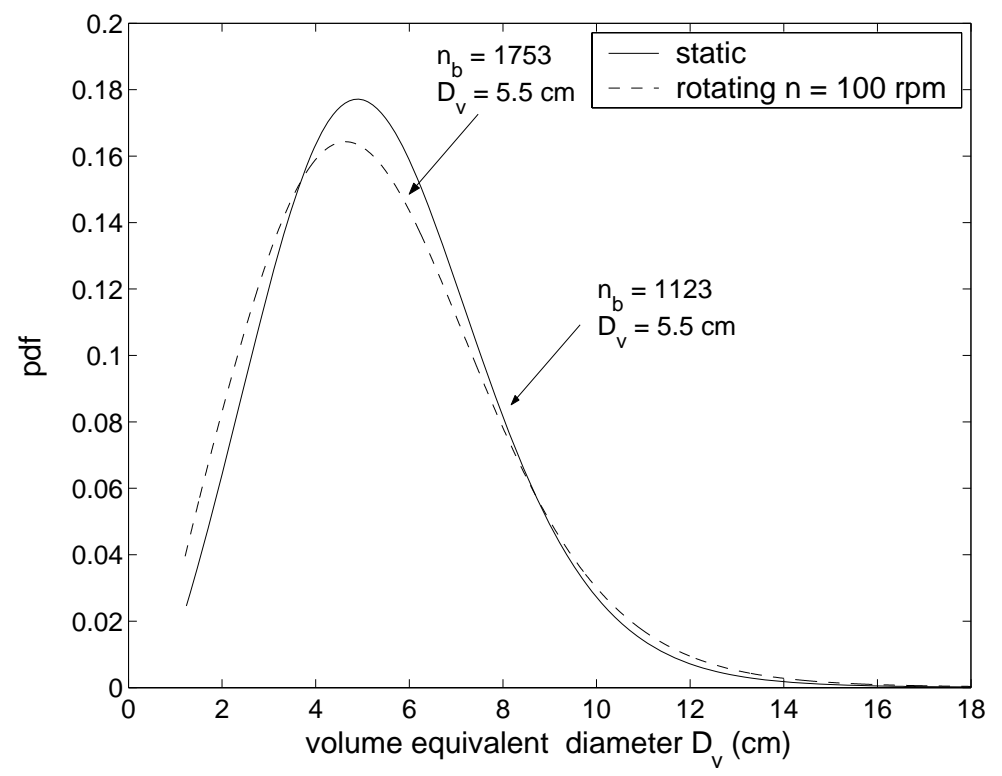

(b)

Fig. 8. PDF of $D_{v}$ at $U=0.57 \mathrm{~m} / \mathrm{s}$ and $z=12.5 \mathrm{~cm}$ for the static $\left(U_{m f, 0}=0.4\right.$ $\mathrm{m} / \mathrm{s})$ and rotating distributor $\left(U_{m f, 100}=0.3 \mathrm{~m} / \mathrm{s}\right)$. (a) $\mathrm{r} / \mathrm{R}=0.5(\mathrm{~b}) \mathrm{r} / \mathrm{R}=0$. The mean of the distribution, $D_{v}$ and the number of bubbles detected by the probe in a 10 min interval are indicated in the plots. 